\title{
TERAPÊUTICA HORMONAL NA CORÉIA DE SYDENHAM
}

\author{
José Geraldo Albernaz * \\ Romualdo José do Carmo **
}

A coréia de Sydenham é geralmente considerada como parte da sindrome da febre reumática. Na sistematização clássica de Jones ${ }^{11}$, ao lado da cardite, da poliartrite, dos nódulos subcutâneos e do eritema, a coréia constitui uma das manifestações principais de febre reumática. Bland e Jones ${ }^{3}$ anotaram a presença de sintomas corêicos em $51 \%$ de 709 casos de febre reumática.

Desde a publicação, em 1949, dos trabalhos de Hench e col.8, 9, a cortisona e o ACTH têm sido empregados no tratamento da febre reumática em suas várias formas. Já em 1950, Massel e col.15 relataram resultados favoráveis com o uso de $\mathrm{ACTH}$ no tratamento de coréia em um paciente de 7 anos; logo em seguida, Bunim ${ }^{4}$ e Kreidberg e col. ${ }^{13}$ referiram bons resultados com o emprêgo do ACTH, enquanto Cutler 5 relatava os mesmos resultados com o uso da cortisona. Entretanto, os vários estudos referentes ao valor terapêutico dêsses hormônioz em casos de coréia não têm chegado a conclusōes uniformes. Aronson e col. ${ }^{2}$, assim como Glasser e Merrit ${ }^{7}$, estão $\epsilon$ tre os primeiros a rclatar resultados negativos com essa modalidade de tratamento.

Segundo Ainger e col.', anormalidades semelhantes, de ordem bioquímica e fisiológica, ocorrem em pacientes com reumatismo poliarticular agudo e coréia de Sydenham, parecendo-lhes ser lógica a eficácia terapêutica do ACTH e da cortisona na coréia de Sydenham, uma vez que êsses medicamentos são considerados eficientes para o reumatismo poliarticular agudo. Dordick e Gluck ${ }^{6}$, empregando a prednisona, considerou-a como substância anti-reumática e anti-inflamatória eficiente; de manejo mais fácil que a cortisona, permitindo emprêgo de doses menores e sem grandes preocupações de dieta, a prednisona, e também a prednisolona, naturalmente obtiveram imediata consideração para o tratamento da coréia.

Trabalho da Clínica Neurológica da Faculdade de Medicina da Universidade de Minas Gerais, apresentado ao I Congresso Latino-Americano de Neurologia, 25 a 28 abril 1957, Buenos Aires, Argentina.

* Livre-Docente de Clinica Neurológica da Faculdade de Medicina da Universidade de Minas Gerais, em exercicio da Cátedra; diplomado pelo American Board of Neurological Surgery; Cheîe do Serviço de Neurologia e Neurocirurgia do Hospital "Felício Roxo" (Belo Horizonte, Minas Gerais).

** Assistente voluntário da Clinica Neurológica da Faculdade de Medicina da Universidade de Minas Gerais (Belo Horizonte, Minas Gerais). 
Como contribuição às publicações anteriores sôbre o assunto, propomonos a relatar 5 casos por nós observados.

\section{MATEIRIAL}

Foram observados 5 pacientes ( 2 meninos e 3 meninas), com idade entre 8 e 15 anos. O critério da escolha dos mesmos baseou-se principalmente na evidência clínica de coréia, pela presença de movimentos involuntários de tipo corêico, com ou sem outras manifestações maiores de febre reumática.

Foram usados esquemas de tratamento à base de cortisona em dois casos e de prednisona nos outros três, sem prejuízo da terapêutica sintomática usual.

\section{OBSERVAC̄ES}

Caso 1 - J. G. S., sexo masculino, 11 anos de idade, branco, internado em 255-1956. Há 2 meses teve inchação e dor nas articulações do tornozelo, joelho e cotovelo, com caráter migratório. O acometimento articular persistiu por 8 dias mais ou menos. Há 10 dias surgiram movimentos involuntários do tipo corêico. Em 1954 já tivera reumatismo e hemicoréia. Exame físico normal. Hemossedimentação: 23 $\mathrm{mm}$ na $1^{\mathrm{a}}$ hora e $44 \mathrm{~mm}$ na $2^{\text {a }}$ hora (Westergreen).

Terapêutica $\epsilon$ evolução - Na primeira semana, foram usados $100 \mathrm{mg}$ de Cortisona durante 3 dias, $75 \mathrm{mg}$ durante 2 dias e $50 \mathrm{mg}$ nos 2 dias restantes; a resposta não foi a desejada. Nova prova de hemossedimentação revelou $16 \mathrm{~mm}$ na $1^{\text {* }}$ hora e $60 \mathrm{~mm}$ na 2 hora. Foi aumentada a dose diária de Cortisona para $200 \mathrm{mg}$, durante 27 dias. A melhora foi progressiva, tendo sido diminuicia a dosc diária para $25 \mathrm{mg}$, durante 30 dias. Nos últimos 20 dias o paciente iá não apresentava sintomas da doença e, ao receber alta, a velocidade de sedimentação das hemácias era de $5 \mathrm{~mm}$ na 1* hora e $10 \mathrm{~mm}$ na 2 " hora. Luminal e Clorpromazina foram usados como medicaçāo sintomática auxiliar. Prescreveu-se tambẻm Penicilina-Benzotina.

Caso 2 - J. H. S., sexo masculino, 9 anos de idade, mulato, internado em 186-1956. Há 2 meses começou a repuxar todo o corpo. Inicialmente, um ou outro repuxão num braço ou numa perna; aos poucos, êstes movimentos se foram acentuando, sendo que há 8 dias pioraram bastante. Seus parentes informam que os movimentos desaparecem durante o sono e o paciente dorme tranqüilo. Atualmente, repuxa os cantos da bôca, mexe com a cabeça, o braço, uma ou outra perna, em sucessão indefinida. Refere ter, também, sensação de picadas e dôres nas pernas. Há 4 dias vem tendo dificuldades para falar. Queixa-se de dor de cabeça de vez em quando. Dispnéia quando anda ou corre. Palpitaçōes, às vêzes. Há 2 anos sentiu pontadas no coração. Queixa-se de crises de aflição, como se houvesse alguma coisa a sufocá-lo, mas não relata dispnéia paroxística noturna. Antecedentes pessoais - Com 1 ano de idade, teve febre alta durante vários dias, diagnosticada como tifo; teve esquistossomose, tendo sido tratado com Fuadina, há 1 ano; àlém disso, coqueluche e sarampo, sem gravidade. Exame físico - Movimentos involuntários do tipo corêico, bilaterais. Aparêlho circulatório: Ictus visivel no $4^{\circ}$ espaço intercostal; pela palpação, frêmito de média intensidade, sistólico, na ponta; ictus intenso, coberto por 3 polpas digitais; pela ausculta: sôpro sistólico rude, com irradiação para a axila esquerda. Pulso radial duro, ritmado, cheio, com 68 batimentos por minuto. Tensão arterial: Mx 110, Mn 65. Dôres à palpação, no hipocôndrio direito, logo abaixo da reborda costal. Figado palpável a um dedo da reborda. Baço não palpável.

Terapêtica e evolũăo — Foram administradas $150 \mathrm{mg}$ de Cortisona durante 4 dias, $100 \mathrm{mg}$ durante 16 dias e $50 \mathrm{mg}$ durante 48 diaz. Melhora clinica 20 dias de- 
pois do inicio da administração da Cortisona; assintomático apćs mais 28 dias. Alta após mais 20 dias de observação. Foram empregados também o Luminal como medicação sintomática auxiliar, e a Penicilina-Benzatina. Neste caso, antes de ser iniciado o uso de Cortisona, fôra administrada Butazolidina, sem resultado, durante 20 dias.

Caso 3 - - M. G. P. S., sexo feminino, 8 anos de idade, branca, examinada no ambulatório em 8-8-1956. Movimentos involuntários bilaterais há 20 dias, mais acentuados nos braços e discretos na bôca. Início insidioso, com piora progressiva, estabilizando-se nos últimos 10 dias. Nada de importante nos antecedentes pessoais. Exame - Exceto quanto aos movimentos involuntários do tipo corêico, os exames fisico e neurológico foram normais. Hemossedimentação: $5 \mathrm{~mm}$ da $1^{\text {a }}$ hora e $16 \mathrm{~mm}$ na $2^{\text {n }}$ hora (Westergreen).

Terapêtica e evoluçio - Prescreveu-se Prednisona, na dose de 7,5 mg por dia. Como tratamento sintomático, deu-se Luminal $(0,04 \mathrm{~g}$ por dia). A paciente foi reexaminada 15 dias após; tinha melhorado bastante; notava-se ainda algum movimento nas mãos. Continuou com a mesma medicação e, em mais 5 dias, ficou assintomática. Após mais 10 dias suspendeu-se a Prednisona e prescreveu-se Penicilina-Benzatina.

Caso 4 - A. L. C., sexo feminino, 15 anos de idade, branca, examinada no ambulatório em 20-9-1955. Há 15 dias foi notado que a paciente estava muito inquieta, com movimentos desordenados, derrubando objetos, esbarrando aqui e ali, beliscando a si própria. Perdeu a vontade de estudar. Ficou mais nervosa. Tem dormido bem, mas custa conciliar o sono. Antecedentes pessoais: Aos 10 anos teve processo de tuberculose pulmonar. Difteria aos 6 anos. Coqueluche, sarampo, cachumba e catapora (brandas). Não há história de reumatismo. Há 3 meses féz tratamento anti-rábico, após mordida de cão desconhecido. Menarca aos 14 anos, com atraso nos últimos 2 meses. Exame - Além dos movimentos coreiformes contínuos, os exames físico e neurológico foram negativos. Hemossedimentação: $12 \mathrm{~mm}$ na $1^{\text {s }}$ hora e $48 \mathrm{~mm}$ na $2^{a}$ hora (Westergreen).

Terapêtica e evolução - Prescreveu-se Precinisona, na dose de 2,5 mg 3 vêzes ao dia e, como medicação auxiliar, Luminal $0,05 \mathrm{~g} 2$ vêzes ao dia. Desapareceu a sintomatologia após 25 dias. A paciente continuou em uso de Prednisona durante mais 15 dias. Penicilina-Benzatina também foi usada.

Caso 5 - M. C. G., sexo feminino, 13 anos de idade, branca, examinada no ambulatório em 27-9-1956. Aos 10 anos de idade, começou a apresentar sinais de coréia: repuxava o rosto, as mãos e as pernas. A primeira crise forte durou uns 15 dias. A seguir, ficaram mais fracos os sintomas, mas não desapareceram completamente. Dessa época para cá, a paciente sofreu mais três crises fortes, durante as quais teve que ficar acamada. A última dessas crises iniciou-se há 10 dias. Geralmente, passa melhor quando toma salicilatos, mas, desta vez, como não houvesse melhora, deixou de tomar êste medicamento. Atualmente, mesmo durante o sono, a paciente não fica quieta. Antecedentes pessoais - Amigdalectomia há 3 anos e apendicectomia há 2 meses. Já teve, há anos, dôres reumatóides, porém sem outros indicios de reumatismo poliarticular agudo. Exume - Movimentos involuntários do tipo corêico, mais acentuados na bôca. Exames físico e ncurológico, no mais, negativos. Discreta hipertrofia do ventriculo esquerdo, sugerida pelo eletrocardiograma. Telerradiografia do coração revelando aumento da área cardíaca, principalmente do ventrículo esquerdo e diâmetro cárdio-torácico de 2,2. Hemossedimentacão: $12 \mathrm{~mm}$ na $1^{*}$ hora e $\mathbf{3 1} \mathrm{mm}$ na $2^{\text {n }}$ hora (Westergrcen).

Terapêtica e evolução - Prescreveu-se Prednisona, na dose de 2,5 mg 4 vêzes ao dia, e, como medicação coadjuvante, uminal $0,10 \mathrm{~g}$ por dia. Além disso, a paciente fêz uso de Penicilina-Benzatina, na cose de $1.260 .600 \mathrm{U}$. de 3 em 3 dias, até completar 6.000.000 U. Depois de 20 dias estava bem melhorada. Notavam-se iigeiros movimentos nos lábios; a mãe referiu-se a alguns movimentos discretos nas 
mãos e pés que não observamos durante a consulta. Prescreveu-se Prednisolona, na dose de 7,5 mg diários, Penicilina-Benzatina na dose de 2.400.000 U., uma vez por mês, e $0,05 \mathrm{~g}$ de Luminal duas vêzes ao dia. Em mais 20 dias a paciente estava assintomática; nessa ocasião suspendeu-se o Luminal, continuando-se o uso de Prednisolona por mais 40 dias.

\begin{tabular}{|c|c|c|c|c|c|c|}
\hline Casos & $\begin{array}{c}\text { Duração da } \\
\text { doença antes do } \\
\text { tratamento } \\
\text { hormonal }\end{array}$ & Trutamento & $m g / d i a$ & $\begin{array}{c}N^{\circ} \text { de } \\
\text { dias }\end{array}$ & $\begin{array}{c}\text { Total } \\
m g\end{array}$ & $\begin{array}{l}\text { Desaparecimento } \\
\text { da movimenta- } \\
\text { ção corêica }\end{array}$ \\
\hline \multirow[t]{5}{*}{1} & 10 dias & Cortisona & 100,0 & 2 & & \\
\hline & & & 75,0 & 3 & & \\
\hline & & & 50,0 & 2 & & \\
\hline & & & 200,0 & 27 & & \\
\hline & & & 25,0 & 30 & 6.675 & 44 dias \\
\hline \multirow[t]{3}{*}{2} & 60 dias & Cortisona & 150,0 & 4 & & \\
\hline & & & 100,0 & 16 & & \\
\hline & & & 50,0 & 48 & 4.600 & 48 dias \\
\hline 3 & 20 dias & Predniosna & 7,5 & 27 & 200 & 20 dias \\
\hline 4 & 15 dias & Predniosna & 7,5 & 40 & 360 & 25 dias \\
\hline \multirow[t]{2}{*}{5} & 10 dias & Prednisona & 10,0 & 20 & 200 & \\
\hline & & Prednisolona & $7, \bar{C}$ & 60 & 450 & 40 dias \\
\hline
\end{tabular}

Quadro 1-Resultados obtidos nos 5 casos.

\section{COMENTARIOS}

Em todos os casos houve desaparecimento completo dos sintomas corêicos, em períodos que variaram de 20 a 48 dias. Nos dois pacientes em que foi usada a cortisona, as doses totais empregadas foram altas, respectivamente, $6.675 \mathrm{mg}$ e $4.600 \mathrm{mg}$; se tivéssemos suspendido a medicação ao alcançarmos doses eqüivalentes às usadas por Aronson, Douglas e Lewis ${ }^{2}$ ou Glasser e Merrit ', certamente teríamos obtido resultados insatisfatórios nesses dois casos. Com o uso da prednisona e da prednisolona, alcançamos resultados bons com doses bem reduzidas e períodos de tratamento menos prolongados.

Schwartzman e col.16 preconizam o emprêgo simultâneo de ACTH e cortisona, de 6 em 6 horas, usados até desaparecimentc dos sintomas, o que 
ocorreu, em sua experiência, em períodos de 7 a 32 dias; êsses autores consideram essa forma de tratamento superior à terapêutica com apenas uma das drogas ou durante prazos limitados. Kroop ${ }^{14}$ chama a atenção para as vantagens de doses elevadas, sugerindo, em média, de 200 a $400 \mathrm{mg}$ diários de cortisona por periodos de 2 a 3 semanas, seguidos de 150 a $200 \mathrm{mg}$ diários por uma ou duas semanas e, depois, por $100 \mathrm{mg}$ diários durante uma semana e mais $50 \mathrm{mg}$ diários por outra semana; o tratamento deve durar em média de 6 a 12 semanas, com doses individualizadas de acôrdo com a gravidade e a evolução da moléstia; com essas dosagens, de 5 casos de coréia, 3 apresentaram melhoras em uma semana.

Kelly 12, em 18 casos de febre reumática aguda, dentre os quais 3 com coréia, obteve resultados dramáticos com a Corticotropina, estabelecendo, como dose diária inicial ótima, a de 1 a 2 unidades internacionais por libra de pêso corporal; êste autor analisa as possíveis causas de insucesso da terapêutica hormonal nas moléstias reumáticas. É óbvio que essas mesmas causas devem prevalecer para a coréia de Sydenham: a) variabilidade da doença no que diz respeito à gravidade e duração de cada uma de suas várias manifestações; b) variação no critério empregado para estabelecer o diagnóstico da doença; c) variação na dosagem das drogas empregadas; d) seleção dos casos, incluindo primàriamente pacientes que exibem predominância de determinada manifestação da doença; e) falta de seleção de pacientes no que diz respeito a ataques iniciais agudos, recidivantes agudos e fase crônica ativa da doença.

$\hat{E}$ sabido que um episódio de coréia de Sydenham pode desaparecer espontâneamente em prazo variável entre 6 a 10 semanas. Entretanto, as recidivas são freqüentes, possivelmente acima de $70 \%$, segundo Ainger e col. ${ }^{1}$.

Nossos casos ficaram assintomáticos em menos de 6 semanas e em nenhum registrou-se recidiva após a terapêutica hormonal, pelo menos até agora, Com o fim de afastar as recidivas, valemo-nos da antibioticoterapia profilática, como sugeriram Bland e Jones ${ }^{3}$, em forma semelhante à preconizada recentemente por Stollerman ${ }^{17}$, que sugere, entre outras alternativas, uma injeção mensal de 1.200.000 U. de Penicilina-Benzatina.

Com a intenção de observar os resultados da terapêutica hormonal na coréia, abstivemo-nos do uso de salicilatos, embora Holt e col." relatem magníficos resultados na febre reumática com o uso da Cortisona associada aos salicilatos.

\section{RESUMO E CONCLUSÕES}

Cinco casos de coréia de Sydenham foram submetidos à terapêutica hormonal com Cortisona, Prednisona e Prednisolona; o fenobarbital e a clorpromazina foram utilizados como medicação sintomática e a Penicilina-Benzatina como profilática. Depois de analisadas as referências bibliográficas, comparados os resultados de v;rios autores, e ajuizados os resultados obtidos em nossos casos, acreditamos poder concluir que: 1) a terapêtica hormonal da coréia de Sydenham, com o uso de ACTH, Cortisona, Prednisona ou Pred- 
nisclona apresenta resultados satisfatórios; 2) a Prednisona e a Prednisolona são os hormônios mais úteis no tratamento da coréia de Sydenham, sendo possível que sua associação aos salicilatos permita obter resultados ainda melhores; 3) o tratamento deve prolongar-se até o ccmpleto desaparecimento doz sintomas, as doses variando de acôrdo com a gravidade e a evolução da moléstia; 4) a terapêutica hormonal deve ser usada sem prejuízo de medicação sintomátice (sedativa) e profilática (antibiótica).

\section{SUMMARY AND CONCLUSIONS}

\section{Hormonal therapy of Sydenhum's chorea.}

Five cases of Sydenham's chorea were treated with Cortisone, Prednisone and/or Prednisolone; Phenobarbital and Chlorpromazine were used as symptomatic and Benzathine-Penicillin as prophylatic medications. Reports of other workers were analyzed and their results compared. It is believed that the following conclusions are justified: 1) hormonal therapy of Sydenham's chorea with ACTH, Cortisone, Prednisone or Prednisolone brings satisfactory results; 2) Prednisone and Prednisolone are the most useful hormones for treatment of Sydenham's chorea, their association with salicylates possibly promising even better results; 3 ) treatment shouid be continued until symptoms disappear and the dosage should be individualized according to the severity and progression of the disease; 4) hormonal therapy should be done together with symptomatic (sedatives) and prophylatic (antibiotics) medications.

\section{REFERENCIAS}

1. AINGER, L. E.; ELY, R. S.; DONE, A. K.; BRILL, A. B.; KELLY, V. C. - $a$ ) Sydenham's chorea: Evidence of abnormal adrenal cortex function. Am. J. Dis. Child., 89:575, 1955; b) Sydenham's chorea: Effects of hormone therapy. Am. J. Dis. Child., 89:580, 1955. 2. ARONSON, N.; DOUGLAS, H. S.; LEWIS, J. M. - Cortisone in Sydenham's chorea: report of 2 cases. J.A.M.A., 145:30, 1951.3 . BLAND, E. F.; JONES, T. D. - The natural history of rheumatic fever: a 20 year perspective. Ann. Int. Med., 37:1006, 1952, 4. BUNIM, J. J. - The clinical effects of Cortisone and ACTH on rheumatic disease. Bull. New York Acad. Med., 27:75, 1951. 5. CUTLER. M. - Cortisone dosage in Sydenham's chorea. J. Med. Soc. New Jersey, 48:226, 1951. 6. DORDICK, J. R.; GLUCK, E. J. - Preliminary clinical trials with Prednisone (Meticorten) in rheumatic diseases: comparative antirheumatic potency; metabolic activity and hormonal properties. J.A.M.A., 158:166, 155. 7. GLASSElR, G. H.; MERRIT, H. H. - Effects of Corticotropin (ACTH) and Cortisone on disorders of the nervous system. J.A.M.A., 148:898, 1952. 8. HENCH, P. S.; KENDALL, E. C.; SLOCUMB, C. H.; POLLEY, H. F. - Effect of hormone of adrenal cortex (17-hydroxydehydrocorticosterone: compound $E$ ) and of pituitary adrenocorticotropin hormone on rheumatoid arthritis. Proc. Staff Meet. Mayo Clin., 24:181, 1949. 9. HENCH, P. S.; SLOCUMB, C. H.; BARNES, A. R.: SMITH, H. L.; POLLEY, H. F.; KENDALL, E. C. - Effects of adrenal cortical hormone 17-hydroxydehydrocorticosterone (compound E) on acute phase of rheumatic fever. Proc. Staff Meet. Mayo Clin., 24:277, 1949.10. HOLT, K. S.; ILLINGWORTH, R. S.; LORBER, J.: RENDLE-SHORT, J. - Cortisone and salicylates in rheumatic fever. Lancet, 2:1144, 1954. 11. JONES, F. D. - The diagnosis of rheumatic fever. J.A.M.A., 126:481, 1944. 12. KELLY, V. C. - Cortico- 
tropin (ACTHI) therapy of initial attacks of acute rheumatic fever in children. Am. J. Dis. Child., 84:151, 1952. 13. KREIDBERG, M. B.; CLEROUX, A. F.; ROSENBERG, I. N. - Clinical evaluation of corticotropin therapy in children. J. Pediat., 39:392, 1951. 14. KROOP, I. X. A. - Treatment of rheumatic fever with large doses of Cortisone. New York J. Med., 54:2699, 1954. 15. MASSEL, B. F.; WARREN, J. E.; STURGIS, A. P.; HALL, B.; CRAIGE, E. - The clinical response of rheumatic fever and acute carditis to ACTH. New England J. Med., 242:641, 1950. 16. SCHWARTZMAN, J.; ZAONTZ, J. B.; LUBOW, H. - Chorea minor. Preliminary report in six patients treated with combined ACTH and Cortisone. J. Pediat., 43:278, 1953.17. STOLlERMAN, G. H. - The Prevention of Rheumatic Fever. W. B. Saunders Co., Filadélfia e Londres, 1957, pág. 133.

Clínica Neurológica da Faculdade de Medicina da Universidade de Minas Gerais Belo Horizonte, Minas Gerais, Brasil. 